\title{
Biofeedback und Neurofeedback: Anwendungsmöglichkeiten in Psychosomatik und Psychotherapie
}

\author{
Biofeedback and Neurofeedback: Applications in Psychosomatic \\ Medicine and Psychotherapy
}

Petra Windthorst ${ }^{1}$, Ralf Veit ${ }^{2}$, Paul Enck ${ }^{1}$, Robert Smolka ${ }^{1}$, Stephan Zipfel ${ }^{1}$, Martin Teufel ${ }^{1}$

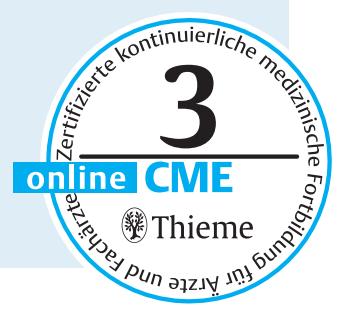

${ }^{1}$ Psychosomatische Medizin und Psychotherapie, Universitätsklinikum Tübingen

2 Institut für medizinische Psychologie und Verhaltensneurobiologie, Universitätsklinikum Tübingen

VNR

2760512015147122499

Bibliografie

Dol http://dx.doi.org/

10.1055/s-0034-1387320

Psychother Psych Med 2015.

65: 146-158

(c) Georg Thieme Verlag KG

Stuttgart · New York

ISSN 0937-2032

Korrespondenzadresse

Petra Windthorst

Universitätsklinikum Tübingen

Psychosomatische Medizin und

Psychotherapie

Osianderstraße 5

72076 Tübingen

petra.windthorst@med.uni-

tuebingen.de

\section{Lernziele}

Der Beitrag soll hinsichtlich der Behandlung mit Biofeedback und Neurofeedback in Psychosomatik und Psychotherapie folgende Aspekte vermitteln:

- Wirkmechanismen

- allgemeine Ziele

- störungsspezifische Anwendungsbereiche

- exemplarischen Behandlungsablauf

Der Leser soll Folgendes übertragen und praktisch anwenden können:

- Beratung von Patienten bzw. Klienten über Behandlungsmöglichkeiten mit Biofeedback und ggf. Indikationsstellung in Abhängigkeit des eigenen Arbeitskontextes,

- Aufzeigen von Chancen und

- Grenzen des Verfahrens in Psychosomatik und Psychotherapie.

\section{Zusammenfassung}

$\nabla$

Die Biofeedbacktherapie in der Anwendung in Psychosomatik und Psychotherapie enthält Elemente der Psychoedukation und der Spannungsreduktion. Sie verfolgt mittels gezielter Beeinflussung von Körperfunktionen das Ziel der Beschwerdelinderung, der Leistungssteigerung und der Stärkung des Selbstwirksamkeitserlebens. Biofeedback einschließlich Neurofeedback ist die direkte Rückmeldung von Körperfunktionen, mit dem Ziel diese Funktionen positiv, d.h. in die gewünschte Richtung, zu beeinflussen. Die Rückmeldung erfolgt vornehmlich visuell oder auditiv. Die Biofeedbacktherapie wird den verhaltenstherapeutisch orientierten Psychotherapieverfahren zugeordnet. Das Neurofeedback ist eine spezifische Form der Biofeedbacktherapie, die explizit auf die Beeinflussung neurophysiologischer Funktionen ausgerichtet ist. Im Folgenden wird vorwiegend auf die Behandlung mit Biofeedback und Neurofeedback im Rahmen von psychosomatischen Krankheitsbildern eingegangen, bei denen sich die Biofeedbacktherapie als effizient erwiesen hat.

\section{Historischer Hintergrund \\ $\nabla$}

Der Zusammenhang von physiologischen Prozessen und emotionalem Erleben wurde bereits Ende des 19. Jahrhunderts vom französischen Neurologen Vigouroux oder dem russischen Physiologen Tarchanoff erforscht [1]. Im deutschsprachigen Raum beschäftigten sich Jung und Wertheimer als eine der ersten mit der Aufzeichnung von Veränderungen des Hautleitwertes in Verbindung mit emotional stark behafteten Begriffen [1]. Pionierarbeit in Verbindung mit Theorien der Stressreaktion und -verarbeitung sowie Entspannungstechniken und deren Auswirkungen auf das vegetative Nervensystem leisteten unter anderem Cannon, Seyle und Jacobson Mitte des 20. Jahrhunderts [2]. Im Verlauf der 1930er-Jahre wurde erstmals der Begriff „Biofeedback“ geprägt. Zur Aufzeichnung von physiologischen Reaktionen im Rahmen von juristischen Belangen wurde Anfang des 20. Jahrhunderts der erste Polygraf (ugs. „Lügendetektor“) entwickelt [3]. Gleichzeitig nahm das Interesse an Möglichkeiten der gezielten Beeinflussung von körperlichen Prozessen zu therapeutischen Zwecken kontinuierlich zu [1, 2]. Erste Neurofeedbackbehandlungen an Epilepsiepatienten fanden in den 70er-Jahren statt $[4,5]$. Hinsichtlich der Studien- und Datenlage wurden Biofeedbackbehandlungen in Bezug auf das periphere Biofeedback insbesondere in den 60er- bis 80er-Jahren intensiv untersucht und haben seither Einzug in den klinischen Alltag erhalten [2]. In aktuellen Studien treten dabei Untersuchungen zum Neurofeedback in den Vordergrund [6].

\section{Was ist Biofeedback? \\ $\nabla$}

Übergeordnetes Ziel der Biofeedback- und Neurofeedbacktherapie in Psychosomatik und Psychotherapie ist die Verbesserung des Zusammenspiels von körperlichen und psychischen Funktionen und schließlich eine Beschwerdelinderung bzw. Leistungssteigerung [7]. Bei den verhaltenstherapeutischen Psychotherapieverfahren, denen 
die Biofeedbacktherapie zugeordnet wird, folgt die Behandlung einem strukturierten, zielorientierten Behandlungsplan, der nach einer Problem- und Verhaltensanalyse auf den Ebenen der physiologischen Reaktion, der Emotion und Kognition auf Veränderungen und Verbesserungen auf einer konkreten Verhaltensebene abzielt [8]. Mittels eines elektronischen Messsystems werden in einem Regelkreis physiologische Prozesse vornehmlich visuell oder auditiv, seltener taktil, zurückgemeldet. Über diese Rückmeldung gelingt eine bewusste Wahrnehmung vormals zumeist unwillkürlich ablaufender körperlicher und psychischer Prozesse und die gezielte Beeinflussung der Parameter wird ermöglicht [7]. Durch diese Rückmeldung, auch im Sinne einer positiven Verstärkung (s.u.) und der folgenden bewussten Wahrnehmung der eigenen Körperreaktionen, wird die gezielte Beeinflussung dieser Parameter ermöglicht. Die simultane Rückmeldung einzelner Parameter erlaubt sofortige Einschätzung und bietet Möglichkeiten der Korrektur ( $\bullet$ Abb. 1) $[9-11]$.

Neurofeedback. Eine besondere Form der Biofeedbacktherapie, als ebenfalls nicht invasives Verfahren, ist das Neurofeedback. Es verbindet kognitive Regulationsmechanismen mit Konzepten der Gehirnstimulation und zielt auf die Beeinflussung der Gehirnaktivität ab. Insbesondere bei Störungsbildern, bei denen Hypo- oder Hyperaktivierungen bestimmter Teile des Kortexes bei der Krankheitsentstehung und -aufrechterhaltung eine zentrale Rolle spielen, kann das Neurofeedback als Therapieangebot infrage kommen. Zunehmende Verbreitung findet dabei das Neurofeedbacktraining mittels Elektroenzephalogramm (EEG) [12, 13].

Die Biofeedbacktherapie stellt die Rückmeldung physiologischer Reaktionen an den Probanden dar, fördert eine bewusste Wahrnehmung körperlicher Prozesse, stößt dadurch Lernprozesse an und ermöglicht so positive Veränderungen auf der Verhaltensebene.

\section{Wirkmechanismen und Ziele der Biofeedbacktherapie \\ $\nabla$}

Beim Erlernen von Verhaltensänderungen mithilfe von Biofeedback spielen Mechanismen der klassischen und operanten Konditionierung (z.B. positive Verstärkung), der Interozeption, der physiologischen Reaktionskontrolle als auch der kognitiven Vermittlung von Lernerfolgen eine Rolle $[10,14]$. Ein Verhalten, das durch extrinsische oder intrinsische Konsequenzen belohnt, d.h. positiv verstärkt wird, wird in Zukunft häufiger ausgeführt.

Holroyd und Kollegen [15] zeigten in einer Studie mit 43 Patienten mit Spannungskopfschmerz und

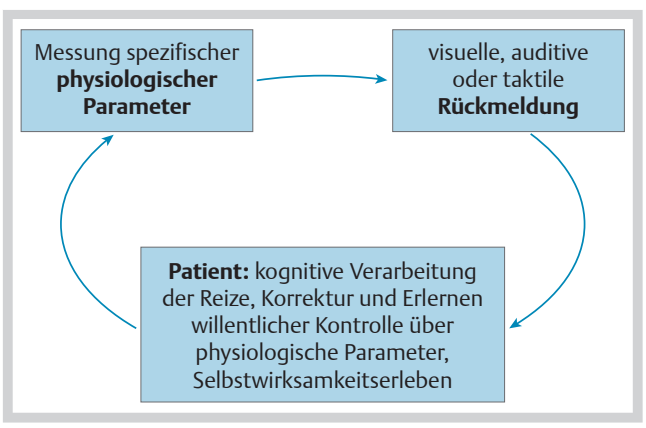

Abb. 1 Regelkreis und Kreislauf des Lernens und Veränderns.

EMG-Feedback, dass die Effektivität der Biofeedbacktherapie insbesondere durch kognitive Veränderungen, die durch die Behandlung angestoßen werden, als ausschließlich durch das Erlernen physiologischer Kontrolle über einzelne Parameter moderiert wird (vgl. auch [16]). Aktuelle Studien gehen in noch größerem Ausmaß von der Bedeutung der kognitiven Komponente, dem Einfluss der wahrgenommenen Kontrolle und der Veränderung des Selbstwirksamkeitserlebens aus. So beschreiben Wheat und Larkin [17] in ihrem kritischen Review-Artikel zum Herzratenvariabilitäts-Biofeedback, dass eine direkte Beeinflussung der Parameter in der Regel nur kurzzeitige physiologische Effekte während der Sitzung und nicht darüber hinaus aufweisen und dennoch langfristig beeindruckende klinische Verbesserungen auftreten ohne nachweisbare signifikante physiologische Veränderungen. Ähnlich benennen Glombiewski et al. [18], dass Biofeedback als psychophysiologische Intervention über die Vermittlung kognitiver Veränderungen, wie die Verbesserung des Selbstwirksamkeitserlebens und das Erlernen von Bewältigungsstrategien, die Effektivität der Biofeedbacktherapie moderiert. Studien mit wirksamem Placebo-Biofeedback (Sham-Biofeedback) als Kontrolle geben ebenfalls Hinweise in diese Richtung u.a. bei den Erkrankungsbildern Fibromyalgie [19], Asthma [20], chronischem Rückenschmerz [21] oder Spannungskopfschmerz [22]. Babu und Kollegen [23] stellten bspw. in ihrer randomisierten kontrollierten doppelblinden Studie zum Einsatz von EMGFeedback und Sham-Feedback bei Patienten mit Fibromyalgie eine Verbesserung in allen Outcome-Variablen in beiden Gruppen fest. Einzelne Unterschiede zwischen den Gruppen zum Vorteil der tatsächlichen Biofeedbackbehandlung ließen sich verzeichnen. Abb. 2 veranschaulicht die genannten Zusammenhänge. Dem Biofeedbacktherapeut kommt dabei eine moderierende und verstärkende Funktion zu.

Hinsichtlich der Neurofeedbacktherapie und ihren Wirkmechanismen wird häufig eine Vermengung unabhängiger und abhängiger Variablen diskutiert, d.h. der Einfluss der Gehirnaktivität auf kognitive Prozesse und umgekehrt der Einfluss kognitiver Aktivität auf zerebrale Prozesse. Micoulaud-Franchi und Kollegen [24] schlagen

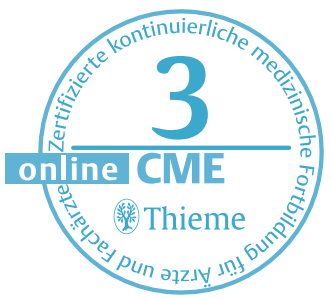


Abb. 2 Modell zur Beschreibung der Wirkmechanismen der Biofeedbacktherapie [15].

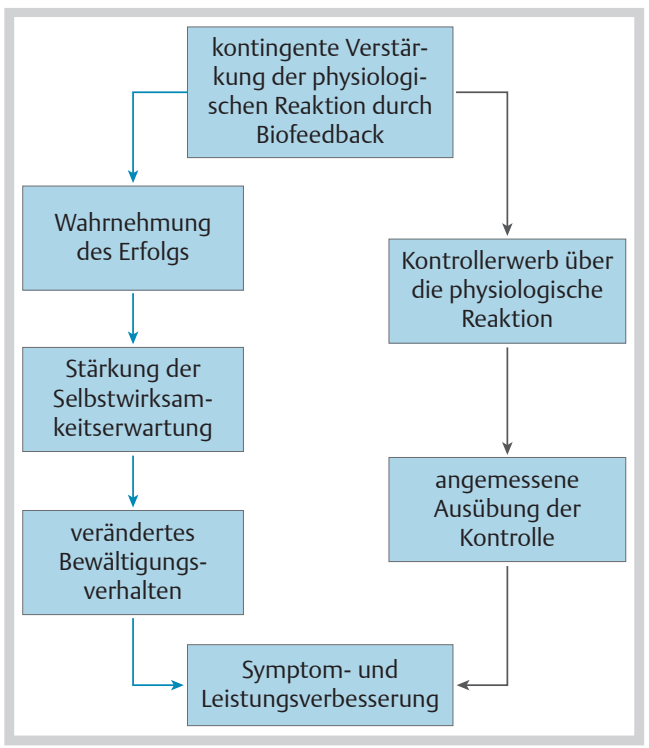

ein Modell vor, das sowohl eine „neuropsychologische“ als auch eine „psychophysiologische“ Herangehensweise und die genauere Extraktion abhängiger und unabhängiger Variablen verdeutlicht, mit dem Ziel kognitive Prozesse und Variablen der Gehirnaktivität besser zu benennen. Darüber hinaus ermöglicht der Einsatz des fMRTFeedbacks den Probanden mit den eigenen kognitiven Prozessen und ihren Effekten auf die Aktivität des jeweiligen Gehirnareals zu experimentieren [24].

Störungsunspezifisches Lernen. Neben der konkreten Beeinflussung einzelner Parameter (s.u.) ist also ein übergeordnetes, störungsunspezifisches Lernen möglich. So erleichtert eine konkrete Rückmeldung physiologischer Prozesse die Veranschaulichung psychosomatischer und somatopsychischer Zusammenhänge und kann einen Beitrag zur Psychoedukation leisten. Wie im obigen Modell von Holroyd et al. in $\bullet$ Abb. 2 dargestellt, kann die Biofeedbacktherapie den Aufbau von Selbstwirksamkeits- und Kontrollerwartungen unterstützen und diese verstärken. Durch die Aufmerksamkeitslenkung auf innere physiologische Veränderungen verbessert sich die Interozeption. Nicht zuletzt kann das Biofeedback ferner die allgemeine Entspannungsfähigkeit verbessern (vgl. auch [1] bzw. [25]).

Die Biofeedbacktherapie erlaubt eine spezifische Einflussnahme auf Parameter und unspezifische Veränderungen in Bezug auf Lernerfahrungen. Dabei ist die Verbesserung des Selbstwirksamkeitserlebens ein bedeutsamer Effekt der Intervention.
Trainingsparameter und Indikationsgebiete

$\boldsymbol{\nabla}$

Neurofeedback

Im Bereich des Neurofeedbacks gibt es unterschiedliche Möglichkeiten der Berechnung von Trainingsparametern, die mit dem EEG erhoben werden. Am häufigsten werden das Frequenzband-Training (Alpha-, Beta-, Theta-, Delta-Wellen), das Training von langsamen kortikalen Potenzialen (Slow Cortical Potentials, SCP) oder das Infra-Low Frequency Training angewendet [26]. Das EEG-Neurofeedbacktraining ist in der klinischen Routine am häufigsten eingesetzt. Vornehmlich im experimentellen Status befinden sich Neurofeedbackverfahren im Bereich der funktionellen Magnetresonanztomografie (fMRT) und Nahinfrarotspektroskopie (fNIRS) (s.u.).

\section{Biofeedback}

Beim Einsatz des Biofeedbacks in der Psychosomatik und Psychotherapie wird in der Regel ausgehend von der vorliegenden Beschwerdesymptomatik gemeinsam mit dem Patienten entschieden, über welchen Parameter und welches Training eine Symptomlinderung erreicht werden soll und kann. Übergeordnete Ziele, wie die Verbesserung der Interozeption oder der allgemeinen Entspannungsfähigkeit, werden dabei ebenfalls berücksichtigt. Die primär zur Verfügung stehenden physiologischen Parameter sind (vgl. $[11,25])$ :

- die elektrische Aktivität der Muskulatur

(Elektromyografie),

- die elektrodermale Aktivität

(Hautleitfähigkeit bzw. Hautwiderstand),

- die kardiale Aktivität

(Herzfrequenz, Herzratenvariabilität),

- die periphere Durchblutung (Blutvolumen,

Pulsvolumenamplitude, Blutdruck, periphere

Durchblutung),

- die Haut- und Körpertemperatur sowie

- die Atemtätigkeit.

Anwendungsbereiche. Die Biofeedbacktherapie wird bei unterschiedlichen Störungen aus dem Bereich der somatischen, der somatopsychischen (auch hirnorganischen) und psychosomatischen Störungen angewendet. In Tab. 1 wird eine Auswahl verschiedener Anwendungsbereiche mitsamt gängiger Trainingsparameter vorgestellt und eine Einschätzung der Effektivität nach derzeitigem Stand sowie in Anlehnung an Martin und Rief [25] sowie Schwartz [27] gegeben. Da die genannten Beschwerdebilder mitunter sehr heterogene Störungsgruppen umschreiben, bisweilen alternative Behandlungsverfahren vorhanden sind und die Wirksamkeitsnachweise sowohl symptomspezifische als auch symptomübergeordnete Variablen berücksichtigen, handelt es sich um relative Empfehlungen.

Kontraindikationen. An erster Stelle der Überlegungen zur Anwendung des Biofeedbacks stehen 
primär jeweils der individuelle Patient und seine Beschwerdekonstellation [25, 27]. Für die Behandlung mit Biofeedback gibt es keine Altersbeschränkung, solange die Rückmeldung von den Probanden kognitiv erfasst werden kann. Bisher sind keine durch Untersuchungen abgesicherten Kriterien über Kontraindikationen festgelegt. Dennoch sollte das Biofeedback besonders vorsichtig angewendet werden bei [27]:

- schweren Depressionen,

- akuter Agitiertheit,

- akutem (prä-)psychotischen Erleben,

- Manie (auch im Rahmen einer bipolaren Störung),

- hochgradiger Zwangsstörung oder

- Gefahr akuter Dissoziation.

Nebenwirkungen. Hinsichtlich der Nebenwirkungen der Behandlungsmethode wird die Biofeedbacktherapie häufig als besonders nebenwirkungsarm beschrieben [7]. Limitierend bleibt, dass viele Biofeedbackstudien Nebenwirkungen nicht systematisch erfasst haben. Landman et al. [28] berichten bspw. in ihrer Studie zum Einsatz des Atem-Feedbacks bei Patienten mit Bluthochdruck und Diabetes mellitus Typ 2 von zwei Patienten, die aufgrund von unerwünschten Nebenwirkungen wie Kurzatmigkeit und Brustschmerzen abbrachen. Hinsichtlich des fMRT-Feedbacks bezeichnen Sulzer und Kollegen [29] den Einsatz des Verfahrens als relativ risikofrei, sofern die Sicherheitsrichtlinien angemessen umgesetzt werden. Hawkinson et al. [30] zeigten in ihrer Studie zur Untersuchung unerwünschter Effekte von fMRT-Scanning und Echtzeit-fMRT-Training, dass kein signifikanter Anstieg von unerwünschten Ereignissen eintrat. Sulzer und Kollegen [29] geben als mögliches Behandlungsrisiko die Induktion einer dysfunktionalen neuronalen Plastizität an, sofern wiederholt dysfunktionale Strategien trainiert werden. Als häufigste berichtete unerwünschte Nebenwirkungen werden mentale Erschöpfung und körperliches Unbehagen benannt, beides Nebenwirkungen, die natürliche Begleiterscheinungen von Anwendungen sind, die hohe Konzentration und minimale Kopfbewegungen erfordern [29]. Entsprechend beschreiben Kravitz et al. [31] als häufigste genannte Nebenwirkung Müdigkeit und Erschöpfung im Rahmen einer Studie zum EEG-Feedback bei Patienten mit Fibromyalgie.

Wirksamkeit. Schoenberg und David [6] fassen in ihrem systematischen Review zu Biofeedbacktherapie bei psychiatrischen Erkrankungen, in den 63 Studien eingeschlossen wurden, zusammen, dass $80,9 \%$ der Studien von gewisser klinischer Verbesserung und $65 \%$ der Studien von statistisch signifikanter $(p<0,05)$ Symptomreduktion standardisierter klinischer Parameter berichten. Dabei war in diesem Kontext der am häufigsten verwendete Trainingsparameter EEG-Feedback. Die am häufigsten untersuchte Störungsgruppe stellten Angsterkrankungen dar. Interessanterweise
Tab.1 Auswahl an Anwendungsbereichen für die Bio- und Neurofeedbacktherapie in Verbindung mit möglichen Trainingsparametern und orientierender Einschätzung der Wirksamkeit nach derzeitigem Stand.

\begin{tabular}{|c|c|c|}
\hline Beschwerdebild & Trainingsparameter & Wirksamkeit \\
\hline Adipositas [32] & $\mathrm{SCL}$ & $(+)$ \\
\hline $\begin{array}{l}\text { Asthma bronchiale [20] und } \\
\text { chronisch obstruktive Lungen- } \\
\text { erkrankung [33] }\end{array}$ & Atmung, EMG, HRV, RSA & $(+)$ \\
\hline chronischer Rückenschmerz [34] & EMG & $(+)$ \\
\hline chronisches Erschöpfungssyndrom & RSA & $(+)$ \\
\hline Depression $[35,36]$ & EEG, HRV & + \\
\hline Enuresis nocturna & Feuchtigkeitssensor & + \\
\hline Epilepsien & EEG & + \\
\hline essenzielle Hypertonie $[28,37]$ & Atmung, BP, Temperatur, RSA & $(+)$ \\
\hline Fibromyalgie [18] & EMG & + \\
\hline generalisierte Angststörung [38] & EEG & + \\
\hline Harninkontinenz & Ballonkatheter, EMG & + \\
\hline $\begin{array}{l}\text { kraniomandibuläre Dysfunktion } \\
\text { [39] }\end{array}$ & EMG & + \\
\hline $\begin{array}{l}\text { Kopfschmerzen vom Spannungstyp } \\
\text { [22] }\end{array}$ & EMG & + \\
\hline Migräne [40] & $\begin{array}{l}\text { EMG, Temperatur, } \\
\text { Vasokonstriktion }\end{array}$ & + \\
\hline Panikstörung [41] & Atmung, $\mathrm{pCO}_{2}$ & + \\
\hline $\begin{array}{l}\text { posttraumatische Belastungs- } \\
\text { störung [42] }\end{array}$ & EEG, EMG, HRV, SCL, Temperatur & $(+)$ \\
\hline Raynaud-Symptomatik & Temperatur & $(+)$ \\
\hline Schlafstörungen & EEG, EMG & + \\
\hline sexuelle Funktionsstörungen & EMG & $(+)$ \\
\hline spezifische Phobie & Herzfrequenz, SCL & $(+)$ \\
\hline Stuhlinkontinenz [43] & Ballonkatheter, EMG & $(+)$ \\
\hline Tinnitus [44] & EMG & $(+)$ \\
\hline
\end{tabular}

$+=$ nachgewiesene positive Effekte im Rahmen von mind. 2 kontrollierten randomisierten Studien; $(+)=$ Hinweise für positive Effekte, nicht ausreichende Studienlage; SCL= Hautleitwert, EMG = Elektromyogramm, HRV= Herzratenvariabilität, RSA=Respiratorische Sinusarrhythmie, BP=Blutdruck, EEG=Elektroenzephalogramm, $\mathrm{pCO}_{2}=$ Kapnometer

führten die Autoren an, dass multimodales Feedback am effektivsten zur Symptomverbesserung beitrug.

Limitierende Aussagen zur Wirksamkeit liegen, u.a. aufgrund fehlender kontrollierter, randomisierter Studien derzeit zur störungsspezifischen Biofeedbackbehandlung von vorwiegend heterogenen Krankheitsbildern wie Essstörungen, Persönlichkeitsstörungen, Schizophrenien und wahnhaften Störungen oder bei intelligenzgeminderten Personen vor [25]. Bei diesen Störungsbildern kann das Biofeedback als zusätzliches Entspannungsverfahren hilfreich sein [32], jedoch genügt es nach jetzigem Kenntnisstand nicht für eine symptomspezifische Behandlung. Insgesamt lässt sich festhalten, dass sich Biofeedbacktherapie bei Erkrankungen, bei denen physiologische Mechanismen nicht unmittelbar an der Entstehung und Aufrechterhaltung der Störung beteiligt sind, weniger nutzbar zeigt, wenngleich sie auch bei diesen Erkrankungen als „Türöffner" zum Verständnis für psychobiologische Zusammenhänge dienen und zur Etablierung einer therapeutischen Beziehung beitragen kann [6].

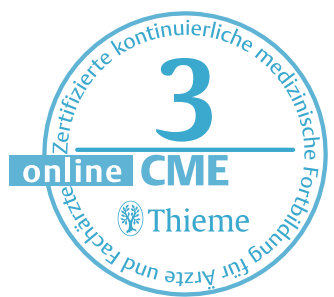


Tab.2 Therapiemanual zur Biofeedbackbehandlung bei chronischem Erschöpfungssyndrom (CFS).

\begin{tabular}{|c|c|}
\hline Sitzung & Inhalt \\
\hline $\begin{array}{l}1 . \\
\text { vertraut machen } \\
\text { mit dem Setting } \\
\text { und Belastungs- } \\
\text { test }\end{array}$ & $\begin{array}{l}\text { - vertraut machen mit Setting und erhobenen Parametern } \\
\text { - individuelle Reaktion bei Belastung bei Einsatz eines mentalen } \\
\text { (Rechentest) und eines individuellen emotionalen Stressors } \\
\text { - Auswertung des Belastungstests, Psychoedukation über biopsy- } \\
\text { chosoziale Zusammenhänge } \\
\text { - abschließend erstmalige direkte Rückmeldung der RSA }{ }^{1} \text { in Form } \\
\text { von Atmung und Pulsfrequenz } \\
\text { - Einführung des (Symptom-/Erschöpfungs-)Tagebuchs }\end{array}$ \\
\hline $\begin{array}{l}2 . \\
\text { experimentieren } \\
\text { mit RSA }\end{array}$ & $\begin{array}{l}\text { - experimentieren mit Erhöhung und Senkung der Atemfrequenz, } \\
\text { Wahrnehmung der Veränderungen in der Herzfrequenz und in } \\
\text { eigenem Erleben } \\
\text { - Wahrnehmung der eigenen Einflussmöglichkeiten } \\
\text { - Einführung des regelmäßigen, eigenständigen Übens zwischen } \\
\text { den Trainingssitzungen im Sinne einer kontinuierlichen „Haus- } \\
\text { aufgabe“ }\end{array}$ \\
\hline $\begin{array}{l}3 . \\
\text { Aufmerksamkeit } \\
\text { und Wahrneh- } \\
\text { mung }\end{array}$ & $\begin{array}{l}\text { - Übung zur Wirkung von Aufmerksamkeitsumlenkung auf die } \\
\text { Wahrnehmung körperlicher Beschwerden² } \\
\text { - Verdeutlichung des Zusammenhangs von Aufmerksamkeitsfo- } \\
\text { kussierung, Bewertung, Emotion und Symptomwahrnehmung } \\
\text { - Integration in ein biopsychosoziales Störungsmodell }\end{array}$ \\
\hline $\begin{array}{l}4 . \\
\text { Erleben von } \\
\text { Selbstwirksamkeit } \\
\text { und Kontrolle un- } \\
\text { ter erleichterten } \\
\text { Bedingungen }\end{array}$ & $\begin{array}{l}\text { - Übung und Vertiefung der eigenen Einflussnahme auf RSA unter } \\
\text { optimalen Bedingungen } \\
\text { - RSA als zusätzliche individuelle Ressource } \\
\text { - Erleben von eigener Kontrolle, Handlungsspielraum und Gestal- } \\
\text { tungsmöglichkeiten }\end{array}$ \\
\hline $\begin{array}{l}5 .-7 . \\
\text { Übung \& Selbst- } \\
\text { wirksamkeitserle- } \\
\text { ben unter zuneh- } \\
\text { mend erschwer- } \\
\text { ten Bedingungen }\end{array}$ & $\begin{array}{l}\text { - Konsolidierung des Erlernten } \\
\text { - Generalisierung sowohl einzelner Biofeedback-Übungen als auch } \\
\text { individueller Veränderungen zur Verbesserung der Erschöp- } \\
\text { fungssymptomatik } \\
\text { - zunehmende Integration in belastende Situationen sowohl } \\
\text { während der Therapieeinheit als auch in therapiefreier Zeit } \\
\text { - Wiederholung der Psychoedukation zu individueller Stressreak- } \\
\text { tion, Aufmerksamkeitsfokussierung, psychophysiologischen } \\
\text { Zusammenhängen und Störungsmodell sowie individuellen } \\
\text { Belastungssituationen } \\
\text { - Erleben von Kontrolle und Selbstwirksamkeit während des Übens }\end{array}$ \\
\hline 5. & $\begin{array}{l}\text { - Zwischenbilanz } \\
\text { - Rückblick über die bisherigen Therapieeinheiten (Stresstest, } \\
\text { Aufmerksamkeit und Symptomwahrnehmung, eigene Einfluss- } \\
\text { möglichkeiten auf vegetative Funktionen, Zusammenhang von } \\
\text { individuellen Stressoren und Erschöpfungssymptomen) } \\
\text { - Intensivierung der Übungen }\end{array}$ \\
\hline 6. & $\begin{array}{l}\text { - Vorbereitung des Therapieendes } \\
\text { - Ziele für die verbleibenden Stunden und Ausblick } \\
\text { - Übung unter erschwerten Bedingungen z. B. hohes Tempo, } \\
\text { häufigerer Aufgabenwechsel, Reduktion der Besprechungszeit, } \\
\text { hoher Übungsanteil }\end{array}$ \\
\hline 7. & $\begin{array}{l}\text { - Übung unter erschwerten Bedingungen mit emotional belasten- } \\
\text { den Situationen }\end{array}$ \\
\hline $\begin{array}{l}8 . \\
\text { Kontrolle, Siche- } \\
\text { rung des Therapie- } \\
\text { erfolges \& Ab- } \\
\text { schluss }\end{array}$ & $\begin{array}{l}\text { - Überprüfung der individuellen Reaktion bei Belastung unter er- } \\
\text { neutem Einsatz eines mentalen (Rechentest) bzw. individuellen } \\
\text { emotionalen Stressors } \\
\text { - individuelle Ressource der gleichmäßigen RSA } \\
\text { - Rückmeldung über den Veränderungsprozess während der } \\
8 \text { Sitzungen }\end{array}$ \\
\hline
\end{tabular}

Multimodale Behandlungsansätze. Hinsichtlich der Wirksamkeitsnachweise und Studienlage ist darüber hinaus festzuhalten, dass Biofeedbackbehandlungen, insbesondere im Bereich der somatoformen und Schmerzstörungen, im klinischen
Alltag häufig im Rahmen multimodaler Behandlungsansätze, z.B. in Verbindung mit Psychotherapie, Entspannungstraining, Physiotherapie oder medikamentöser Therapie erfolgreich angewendet werden. Ein spezifischer Effekt einzelner Behandlungsmodule kann dadurch nicht mehr eindeutig verifiziert werden $[18,25]$.

Die Auswahl eines Trainingsparameters erfolgt entsprechend der zugrunde liegenden individuellen Beschwerdesymptomatik. Zahlreiche Wirksamkeitsnachweise für einzelne Störungsbilder liegen vor. Biofeedbacktherapie wird dabei häufig im Rahmen multimodaler Behandlungsansätze eingesetzt.

\section{Therapeutisches Vorgehen und Sitzungs- ablauf}

\section{$\nabla$}

Eine Biofeedbackbehandlung und eine Biofeedbacksitzung folgen in der Regel einem strukturierten Ablauf. Eine Biofeedbackbehandlung umfasst mind. 4 und bis zu 20 Sitzungen. Für eine suffiziente Behandlung mittels Neurofeedback ist die 2-3-fache Sitzungsanzahl keine Seltenheit $[1,8]$.

\section{Behandlungsphasen einer Biofeedback- behandlung}

Der Verlauf einer Biofeedbackbehandlung lässt sich in 3 Phasen gliedern.

Diagnostische Phase. Der Beginn stellt eine diagnostische Phase in Verbindung mit einem Test zur Bestimmung des physiologischen Grundniveaus und zur Messung der Reagibilität unter Belastung dar („Reaktionstest“). In dieser Phase werden zusätzlich das Behandlungsrational vermittelt und Zielparameter festgelegt.

Trainingsphase. In der Trainingsphase selbst werden konkrete Strategien zur Kontrolle des Zielparameters vermittelt. Mithilfe von Schwellenwerten kann im Sinne der operanten Konditionierung, das Erreichen von Zielen und Zwischenzielen positiv verstärkt werden. Mit zunehmenden Kontrollfähigkeiten wird der Schwierigkeitsgrad der Übungen erhöht und die Übungsbedingungen werden variiert.

Transferphase. In der 3. Phase steht der Transfer in den Alltag und die Generalisierung des Erlernten im Vordergrund. Hier wird das Training in relevanten Alltagssituationen gefördert und die direkte Rückmeldung sukzessive ausgeblendet [25]. Beispiel. Die Abfolge verschiedener Behandlungsphasen und -inhalte wird in $\bullet$ Tab. 2 am Beispiel eines Manuals zur Biofeedbackbehandlung bei chronischem Erschöpfungssyndrom (CFS) dargestellt. Die Probandinnen erhielten eine Rückmeldung über ihre respiratorische Sinusarrhythmie (RSA), d.h. ihre Pulsfrequenz und Atmung. Die RSA bzw. Herzratenvariabilität (HRV) spiegelt verschiedene Organsysteme wider, die bei guter Syn- 
chronisation ein suffizientes Level kardiovaskulärer Aktivität aufrechterhalten [17]. Unter dauerhafter Belastung und bei Stresserleben ist diese individuelle, physiologische Reaktion (Stressantwort) häufig dysreguliert. Dies kann zu physiologischen und/oder psychischen Beeinträchtigungen führen [45 - 48]. Umgekehrt kann die Verbesserung der HRV die Stressbewältigung stärken und die Risiken bzw. die Intensität von stressassoziierten Erkrankungszuständen reduzieren $[17,49,50]$.

Das eigenverantwortliche Üben im Alltag ist fester Bestandteil einer Biofeedbackbehandlung und kann in vielen Fällen durch Einsatz portabler Biofeedbackgeräte unterstützt und intensiviert werden [25]. Nestoriuc et al. [40] konnten im Rahmen von Biofeedbackbehandlung bei Patienten mit Migräne die positiven Effekte von begleitendem Training zu Hause eindrücklich belegen. Das Führen eines therapiebegleitenden Symptom- und Übungsprotokolls erweist sich sowohl zur Identifikation von individuellen Belastungssituationen als auch von Ressourcen und Phasen mit reduzierter Symptomatik als hilfreich. Es dient der Besprechung des Verlaufs und der Entwicklungsschritte zwischen den Sitzungen [52].

Sitzungsablauf. Eine einzelne Therapiesitzung untergliedert sich in der Regel in einen Rückblick auf die vorherige Sitzung und die Selbstbeobachtungsaufgabe, dem Anlegen der spezifischen Messfühler und einer Baseline-Messung. Im Weiteren erfolgen Übungseinheiten und zwischenzeitliche Entspannungsphasen im Wechsel. Abschluss stellt der Rückblick über die erfolgte Sitzung und die Vergabe einer konkreten Übungs-/ Selbstbeobachtungsaufgabe bis zur nächsten Sitzung dar [53].

Eine Biofeedbackbehandlung stellt ein strukturiertes, ziel- und lösungsorientiertes Vorgehen in einem in der Regel umschriebenen Trainingszeitraum mit Diagnostik, Training sowie Alltagstransfer dar. Eigenständiges Üben ist dabei zentraler Bestandteil.

\section{Neurofeedback - von der Forschung in die Praxis \\ $\nabla$}

Im Gegensatz zum peripheren Biofeedback zielt das Neurofeedback auf die Beeinflussung zentraler Strukturen ab. Das Neurofeedbacktraining kann über ein Elektroenzephalogramm (EEG), funktionelle Magnetresonanztomografie (fMRT) oder funktionelle Nahinfrarotspektroskopie (fNIRS) erfolgen. Die beiden letzteren Verfahren sind vorwiegend Gegenstand aktueller Forschungsbemühungen wohingegen das EEG-Training bereits in die klinische Anwendung integriert ist.

\section{Elektroenzephalogramm (EEG)}

ADHS. Das EEG-Training ist das am weitesten verbreitete Neurofeedbackverfahren. Bei Kindern mit Aufmerksamkeitsdefizit-/Hyperaktivitätssyndrom (ADHS) wurde festgestellt, dass das Spontan-EEG gegenüber verhaltensunauffälligen Kindern eine erhöhte Theta-Aktivität (Frequenzbereich $4-7 \mathrm{~Hz}$ ) bei gleichzeitig geringerer Beta-Aktivität $(14-40 \mathrm{~Hz})$ aufweist [54]. Neben den Abweichungen im Spontan-EEG wurde auch eine geringere Amplitude bei den langsamen kortikalen Potenzialen beobachtet [55]. Die Veränderungen im EEG lassen sich durch eine dysfunktionale Regulation neuronaler Verarbeitungsprozesse erklären [56]. Basierend auf diesen neurophysiologischen Beobachtungen wurden bereits zu Beginn der 1990er-Jahre die ersten Neurofeedbacktherapien bei ADHS durchgeführt, bei denen die betroffenen Kinder die Beta-Aktivität erhöhen und die Theta-Aktivität erniedrigen sollten. In den letzten Jahren wurden vermehrt die langsamen kortikalen Potenziale für das Training verwendet. Die Kinder sollen dabei versuchen, die elektrische negative Potenzialänderung zu erhöhen. Metaanalysen konnten zeigen, dass das Neurofeedback bei ADHS im Kindes- und Jugendalter klinisch wirksame Effekte in den Symptombereichen Unaufmerksamkeit und Impulsivität aufweist [57]. Eine aktuell laufende multizentrische Studie soll die klinische Wirksamkeit des Neurofeedbacktrainings an einem größeren Kollektiv überprüfen [58].

Epilepsie. Ein weiteres Störungsbild, bei dem bisher die Neurofeedbacktherapie erfolgreich eingesetzt wurde, sind Epilepsien. Tan et al. [59] haben in einer Metaanalyse 10 Studien untersucht und konnten eine bedeutende Reduktion der Anfallshäufigkeit durch das Neurofeedbacktraining berichten. Interessant ist in diesem Zusammenhang, dass die Patienten in diesen Studien allesamt therapieresistent waren, d.h. auf die antikonvulsive Medikation nicht ausreichend ansprachen.

Schlafstörungen. Neben ADHS und Epilepsien wurden EEG-basierte Neurofeedbackverfahren insbesondere zur Behandlung von Schlafstörungen erfolgreich eingesetzt [60]. Bei anderen Störungsbildern gibt es nur vereinzelte Studien, die zwar die prinzipielle Machbarkeit des Therapieansatzes bestätigen konnten, aufgrund fehlender kontrollierter Studien jedoch keine aussagekräftigen Schlussfolgerungen erlauben.

\section{Funktionelle Magnetresonanztomografie (fMRT)}

Die fMRT hat sich in den letzten Jahren zu einer der bedeutendsten Untersuchungsmethoden in den Neurowissenschaften entwickelt. Hiermit kann die neuronale Aktivität in bestimmten Gehirnregionen über lokale Veränderungen der Blutoxygenierung indirekt erfasst werden. Die Technik hat sich in den letzten Jahren so weit entwickelt, dass die fMRT-Ergebnisse bereits kurz

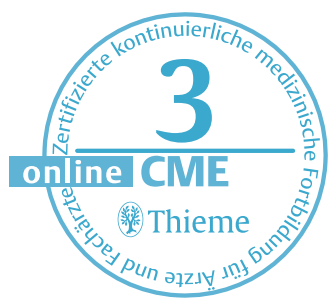


Tab.3 Anwendungsbereiche und mögliche Trainingsparameter einzelner Neurofeedbackverfahren unter Berücksichtigung ihrer Vor- und Nachteile.

\begin{tabular}{|c|c|c|c|}
\hline Verfahren & Anwendungsbereiche & Training & Vor- $(+)$ und Nachteile (-) \\
\hline $\begin{array}{l}\text { EEG } \\
\text { vorwiegend klinischer } \\
\text { Einsatz }\end{array}$ & $\begin{array}{l}\text { Angststörungen } \\
\text { Depression } \\
\text { ADHS } \\
\text { fokale Epilepsie } \\
\text { Schlafstörungen }\end{array}$ & $\begin{array}{l}\text { SMR }(12-15 \mathrm{~Hz}) \\
\text { Beta }(16-20 \mathrm{~Hz}) \\
\text { Theta }(4-7 \mathrm{~Hz}) \\
\text { SCP }\end{array}$ & $\begin{array}{l}\text { + standardisierte Protokolle } \\
\text { + ökologische Validität } \\
\text { + klinische Studien } \\
\text { + mobil/flexibel } \\
\text { + preisgünstig } \\
\text { + zeitliche Verzögerung } \\
\text { - unspezifisch } \\
\text { - lange Vorbereitungszeit }\end{array}$ \\
\hline $\begin{array}{l}\text { fMRT } \\
\text { vorwiegend experi- } \\
\text { menteller Status }\end{array}$ & $\begin{array}{l}\text { psychische und neuro- } \\
\text { logische Erkrankungen }\end{array}$ & $\begin{array}{l}\text { zerebraler Blutfluss } \\
\text { einzelne Regionen } \\
\text { Netzwerk } \\
\text { Konnektivität }\end{array}$ & $\begin{array}{l}\text { + räumlich lokalisiert } \\
\text { + subkortikal } \\
\text { + spezifisch } \\
\text { - teuer } \\
\text { - fehlende Flexibilität } \\
\text { - keine klinische Studien }\end{array}$ \\
\hline $\begin{array}{l}\text { fNIRS } \\
\text { vorwiegend experi- } \\
\text { menteller Status }\end{array}$ & $\begin{array}{l}\text { ADHS } \\
\text { Schlaganfall }\end{array}$ & zerebraler Blutfluss & $\begin{array}{l}\text { + mobil } \\
\text { + kurze Vorbereitungszeit } \\
\text { + weniger artefaktanfällig } \\
\text { - nur kortikale Areale } \\
\text { - geringere räumliche Auflösung } \\
\text { - relativ neue Technik }\end{array}$ \\
\hline
\end{tabular}

ADHS=Aufmerksamkeitsdefizit-/Hyperaktivitätsstörung, SMR = sensomotorischer Rhythmus, SCP=Slow Cortical Potentials (langsame kortikale Potenziale), EEG = Elektroenzephalogramm, fMRT = funktionelle Magnetresonanztomografie, fNIRS= funktionelle Nahinfrarotspektroskopie.

nach der Aufnahme zur Verfügung stehen und somit in Echtzeit weiterverarbeitet werden können. Insbesondere subkortikal gelegene Areale, wie z. B. die Amygdala oder das ventrale Striatum, die bei vielen psychiatrischen und emotionalen Erkrankungen dysfunktionale neuronale Aktivität aufweisen, können für das Echtzeit-fMRT-Feedbacktraining genutzt werden.

Bei gesunden Probanden konnte wiederholt gezeigt werden, dass die Kontrolle der metabolischen Gehirnaktivität zu Verhaltensänderungen führt, die in direktem Zusammenhang mit der funktionellen Einbettung des Zielareals bzw. des neuronalen Netzwerks stehen. Bisherige Studien konnten u.a. belegen, dass das Training zu Leistungsverbesserungen in den Reaktionszeiten [61], in der prosodischen Identifikation [62], der emotionalen Bewertung [63] und der visuellen Erkennungsleistung $[64,65]$ führte und den entsprechenden Kontrollbedingungen (Sham-Feedback) überlegen war.

Anwendungsbereiche. Nach ersten vielversprechenden Trainingsergebnissen bei chronischen Schmerzpatienten [66] folgten in den letzten Jahren einige Machbarkeitsstudien (Proof of Principle Study), die Patienten mit Tinnitus [67], Morbus Parkinson [68] und Nikotinabhängigkeit [69] untersucht haben. In allen Studien lernten die Patienten die ausgewählten Areale erfolgreich zu regulieren und in den meisten Fällen konnten auch positive Verhaltenseffekte ausgemacht werden.

Linden et al. [70] verwendeten das Training erfolgreich bei Patienten mit Major Depression. In dieser Studie sollte während des Trainings die Aktivität in Arealen, die mit positiven Emotionen verbunden sind, erhöht werden. Es zeigte sich, dass es in der Experimentalgruppe im Vergleich zu einer Kontrollgruppe, die positive mentale Vorstellungen ohne Feedback praktizierte, zu einer signifikanten Abnahme der depressiven Symptomatik kam. Diese Ergebnisse sind beeindruckend, wenn man bedenkt, dass die meisten Studien nur einen bis wenige Trainingstage andauerten. Die Überprüfung der Nachhaltigkeit der Ergebnisse ist weitere Forschungsaufgabe.

\section{Funktionelle Nahinfrarotspektroskopie (fNIRS)}

Die fNIRS ist ein Verfahren, mit dessen Hilfe Änderungen des oxygenierten und desoxygenierten Hämoglobins in kortikalen Arealen erfasst werden können. Erste Untersuchungen mit dieser Technik wurden bereits erfolgreich bei Angsterkrankungen, Essstörungen, Persönlichkeitsstörungen und Substanzmissbrauch durchgeführt [71]. Das Verfahren ist zum einen erheblich kostengünstiger als fMRT-basierte Anwendungen und zum anderen kann fNIRS räumlich und zeitlich flexibel eingesetzt werden. Dadurch könnte der Einsatz auf Patienten ausgedehnt werden, bei denen Kontraindikationen bestehen oder die Behandlungen vor Ort z.B. am Patientenbett durchgeführt werden müssen. Insbesondere Trainingsprotokolle, die einem vorgegebenen Zeitplan folgen müssen, können flexibler angepasst werden. Die Technik erlaubt jedoch nur die Erfassung kortikaler hämodynamischer Aktivierungsmuster bis zu einer Tiefe von 2 bis $3 \mathrm{~cm}$, sodass subkortikale Strukturen nicht erfasst und somit für diese Art des Neurofeedbacktrainings nicht genutzt werden können. Erste Ergebnisse einer fNIRS-Neurofeedbackstudie bei Kindern mit ADHS-Sympto- 
matik sind vielversprechend [71]. Insbesondere bei Störungen bei denen die Selbstregulation präfrontaler Gehirnbereiche trainiert werden soll (z.B. bei ADHS), kann dieses Verfahren angewandt werden. Eine Studie mit Schlaganfallpatienten wurde bereits erfolgreich realisiert [72]. Zukünftige Weiterentwicklungen in der Aufnahmetechnik, Artefaktkorrektur und Datenanalyse werden die Sensitivität und Spezifität des NIRSSignals verbessern. Vor- und Nachteile der einzelnen Neurofeedbackverfahren sind in $\bullet$ Tab. 3 zusammenfassend dargestellt.

Das Neurofeedback bietet die Möglichkeit durch Training die Gehirnaktivität gezielt zu regulieren. Die Selbstregulation neuronaler Aktivität führt zu Verhaltensmodifikationen, die in direktem Zusammenhang mit den veränderten neurophysiologischen Prozessen stehen.

\section{Zusammenfassung und Ausblick}

Die Neuro- und Biofeedbacktherapie sind nicht invasive Verfahren, die im vorliegenden Artikel in ihrer Anwendung im Bereich somatopsychischer, psychosomatischer und somatischer Erkrankungen betrachtet wurden und dort sowohl mit dem Ziel der Beschwerdereduktion als auch der Leistungsverbesserung sowie Verhaltensmodifikation eingesetzt werden können. Der Einsatz von Biofeedbacktherapie in anderen Disziplinen wie der Rehabilitations- und Sportmedizin zur Leistungssteigerung bzw. -wiederherstellung [73], wurde an dieser Stelle nicht berücksichtigt. Versorgung. Der Einsatz der Biofeedbacktherapie in der Psychosomatik und Psychotherapie kann als ein diagnostisches und therapeutisches Verbindungsstück zwischen physiologischem und psychischem Geschehen gesehen werden. Hinsichtlich der aktuellen Versorgungsstrukturen liegen bisher keine genauen Zahlen vor. Dabei ist die Versorgung im (teil-)stationären psychotherapeutischen und psychosomatischen Sektor derzeit noch deutlich besser gewährleistet als im ambulanten Rahmen, in dem sich große Versorgungslücken auftun [25, 74]. Hierbei gilt es die Anzahl und Erreichbarkeit von qualifizierten Biofeedbacktherapeuten weiterhin zu erhöhen. Bisher gibt es in Deutschland noch keinen einheitlichen Standard in der Neuro- und Biofeedbackausbildung [26]. Erschwerend kommen die fehlenden Vergütungsmöglichkeiten und fehlende Entlohnung erhöhter Investitions- und Verbrauchskosten im ambulanten psychotherapeutischen Sektor hinzu [25].

Forschungsaufgaben. Trotz nachgewiesener Wirksamkeit von Neuro- und Biofeedbacktherapie besteht weiterhin spezifischer Forschungsbedarf sowie ein Bedarf zur Verbesserung der methodischen Herangehensweise und der Studien-

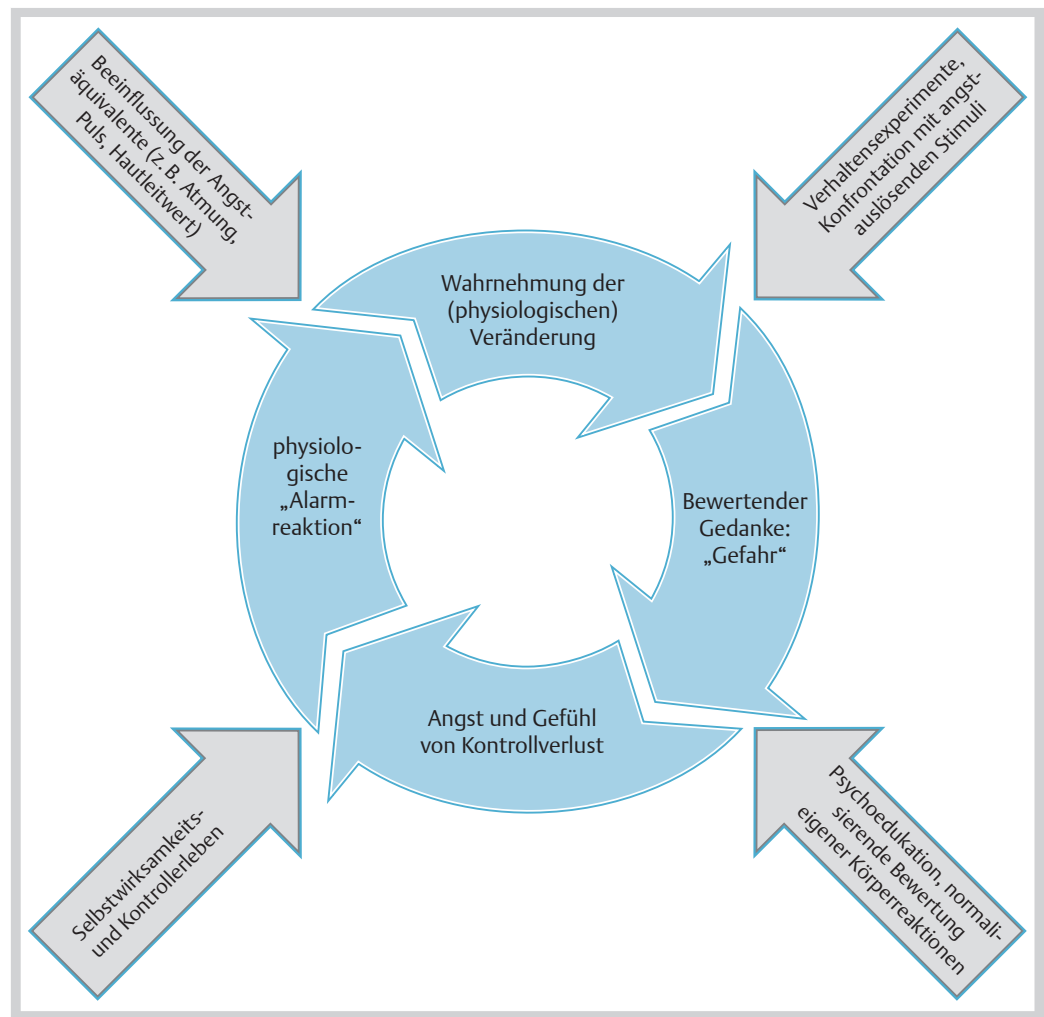

Abb.3 Mögliche Ansatzpunkte (blau) von Biofeedbackbehandlungen bei Patienten mit Angsterkrankungen und somatoformen Störungen mit ausgeprägt ängstlicher Komponente [76].

qualität. So berichten Schoenberg und Davis [6], dass bei $20,6 \%$ der in ihrem aktuellen systematischen Review eingeschlossenen 63 Studien zu Biofeedbacktherapie bei psychiatrischen Erkrankungen eine Kontrollgruppe fehlte und nur die Hälfte der Studien (50,8\%) eine Kontrollgruppe und eine Randomisierung aufwiesen. Auch konkrete Angaben zu Sitzungsanzahl, Intensität/Dauer der einzelnen Sitzung, Kombination von Biofeedbackmodalitäten, begleitender Medikation oder Anwendung eines (bestehenden standardisierten) Protokolls und Angaben zur Störungsspezifität bzw. zur Spezifität der einzelnen Parameter können Rückschlüsse auf die spezifische Wirksamkeit des Verfahrens erlauben [6]. Weitere inhaltliche Forschungsfragen können sich intensiver mit der Anwendung des Biofeedbacks und Neurofeedbacks in Zusammenhang mit und im Vergleich zu etablierten Verfahren (z.B. kognitiver Verhaltenstherapie oder pharmakologischer Therapie) beschäftigen $[18,29,75]$. Hier ist ebenso die Fragestellung des Mehrgewinns von kognitiver Verhaltenstherapie inklusive Biofeedbackbehandlung gegenüber kognitiver Verhaltenstherapie alleine zu überprüfen, was bspw. Glombiewski et al. [34] an Patienten mit chronischem Rückenschmerz untersuchten und zu differenzierten Ergebnissen kamen. Darüber hinaus kann in zukünftigen Studien ein wichtiges Ziel die Aufklärung des Effekts von Selbstwirksamkeits- und Kontrollerleben im Sinne von Moderatorvaria-

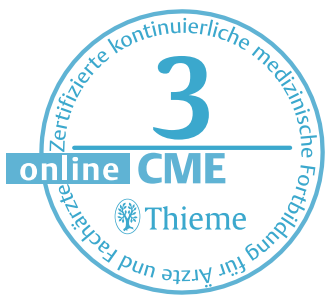


Fallbeispiel

Integration von Biofeedback in einen Gesamtbehandlungsplan bei einem Patienten mit somatoformer und ängstlicher Symptomatik

\section{Anamnese}

Herr M., ein 23-jähriger, in fester Partnerschaft lebender Student, stellte sich aufgrund von Schmerzen und Druckgefühl im Bereich des Herzens und der Brust vor. Die Symptomatik habe vor einem Jahr nach einem Wechsel von Studiengang und Wohnort begonnen. Er verspüre mehrmals täglich ein „Stechen im Herzen und Herzrasen“. Er habe Angst, er könne an einem Herzinfarkt versterben. Er prüfe mind. 1-mal stündlich seinen Puls, um zu kontrollieren „ob noch alles in Ordnung“ sei. Das Einschlafen sei gestört, da er seinen Herzschlag im Liegen und bei Ruhe besonders stark wahrnehme. Morgens wache er „erschöpft und gerädert“ auf. Inzwischen vermeide er körperliche Betätigung, da dadurch die Beschwerden stärker würden. Sein Hobby, das Fußballspielen, habe er aufgegeben und sich sozial zurückgezogen. Das Studium versuche er fortzuführen, wobei er zunehmend Veranstaltungen absage, da er sich „unwohl“ fühle und den Eindruck habe, sein Herz halte der Belastung nicht mehr stand. Das Bestehen des jetzigen Semesters sei ungewiss. Er lebe zusammen mit seiner Partnerin in einer kleinen Wohnung. Seine Partnerin kümmere sich sehr um ihn und habe ihr anstehendes Auslandssemester aktuell verschoben. Der Kontakt zu den Eltern wird weitgehend als gut beschrieben. Vonseiten der somatischen Medizin gebe es keinen Hinweis auf eine organpathologische Genese seiner Beschwerden. Er traue dieser Einschätzung jedoch nicht, sodass er sich wiederholt bei verschiedenen Ärzten kardiologisch, pulmonal, gastroenterologisch und immer wieder hausärztlich vorgestellt habe. Eine in der Pubertät stattgehabte Myokarditis sei vollständig ohne Residuen ausgeheilt. Aktuell nehme er keine Medikamente ein. Zwischenzeitlich habe er abends „zur Beruhigung 1 -2 Bier getrunken“. Dies habe jedoch keine dauerhafte positive Wirkung gehabt, sodass aktuell der Konsum von Alkohol und anderen Genussmitteln verneint wird. Familienanamnestisch berichtet Herr M. vom Versterben seiner Großmutter an Herzinfarkt vor 2 Jahren.

\section{Diagnose}

Diagnose bei stationärer psychosomatischer Aufnahme: somatoforme autonome Funktionsstörung des kardiovaskulären Systems (ICD-10: F45.30).

\section{Therapie}

Eingebettet in ein stationäres, multimodales, psychosomatisches Behandlungssetting wurde das Biofeedback als zusätzliches Verfahren bereits während der gemeinsamen Erarbeitung eines biopsychosozialen Störungsmodells sowohl unter Berücksichtigung lerngeschichtlicher, auslösender als auch aufrechterhaltender Krankheitsfaktoren in der diagnostischen Phase angewendet. Im Belastungstest zu Beginn der Biofeed- backbehandlung konnten psychoedukativ Zusammenhänge zwischen emotionaler Belastung (sozialer und leistungsbezogener Stressor) und physiologischen Reaktionen erklärt werden. Die Auswahl der Übungsparameter (respiratorische Sinusarrhythmie, RSA) erfolgte anhand des Kardinalsymptoms, d.h. des „Herzrasens“ und der „Herzbeschwerden“ als auch orientiert am Zielzustand einer verbesserten psychovegetativen Regulation und Entspannung. Herrn M. wurden folglich parallel Pulsfrequenz als Konfrontations- und Kontrollparameter als auch Atemkurve als direkter Übungsparameter visuell auf einem Monitor zurückgemeldet. Dies stellte anfangs eine Konfrontation im verhaltenstherapeutischen Sinne dar, da die Aufmerksamkeitslenkung auf das Hauptsymptom zunächst in Verbindung mit den Gedanken „da ist etwas nicht in Ordnung“ zu einer Beschleunigung der Pulsfrequenz führte. Dies konnte psychoedukativ aufgegriffen und in ein Modell der Angstentstehung und -aufrechterhaltung integriert werden ( $\bullet$ Abb.3). In Anlehnung an das „Teufelskreismodell der Angst“ von Margraf und Schneider [76] werden dabei spezifische Wirkmechanismen und Ansatzpunkte von Biofeedbacktherapie bei Erkrankungen mit ausgeprägt ängstlicher Komponente zusammengefasst (vgl. auch [52, 77]).

In Verbindung mit der Kontrolle über seine Atmung (gleichförmiges, tiefes Atmen) und der parallelen Rückmeldung von Atmung und Puls konnte Herr M. lernen, dass ein wellenförmiger Verlauf der Pulsfrequenz in Verbindung mit dieser gleichmäßigen, langsamen Atmung ein normaler Prozess ist (RSA) und konnte im Therapieverlauf günstig habituieren. Mit zunehmender Kontrolle über Atmung und Puls gelang es dem Patienten vermehrt in einen Entspannungszustand zu gelangen und seine Grundanspannung, im Sinne der psychovegetativen Aktivierung, zu reduzieren. Ein Gefühl erhöhter Selbstwirksamkeit gegenüber zuvor als unkontrollierbar erlebten Reaktionen stellte sich ein. Eingebettet in das multimodale Behandlungssetting, in dem der Patient unterstützt wurde, sein Sicherheitsverhalten zu reduzieren, soziale Kompetenzen zu stärken und eine berufliche und private Perspektive zu entwickeln, konnte das Biofeedback als konkretes übendes Verfahren der Psychoedukation, der Angstkonfrontation sowie der Entspannung dienen. Herr M. erhielt während seines 6-wöchigen stationären Aufenthalts 8 Biofeedbacksitzungen, von jeweils 30-minütiger Dauer. Zu Beginn fanden die Sitzungen 2-mal pro Woche statt, die letzten 3 Sitzungen wurden ausschleichend verteilt. In Anlehnung an das „Teufelskreismodell der Angst“ von Margraf und Schneider [76] werden in $\bullet$ Abb. 3 spezifische Wirkmechanismen und Ansatzpunkte von Biofeedbacktherapie bei Erkrankungen mit ausgeprägt ängstlicher Komponente nochmals zusammengefasst (vgl. auch [52, 77]). blen sein [18]. Mittelfristig ist dabei von zentraler Bedeutung standardisierte Trainingsprotokolle zu etablieren [6], Nachfolgeuntersuchungen zur Überprüfung von Langzeiteffekten durchzuführen und die Effektgrößen zu bestimmen [29]. Langfristig müssen randomisierte kontrollierte und Multicenterstudien folgen, um den Einsatz in der klinischen Praxis sowohl im ambulanten als auch im stationären Kontext weitergehend zu untersuchen.

In der Praxis. Die Anwendung des Verfahrens ist, sobald die Ausrüstung und geschulte Behandler vorhanden sind, leicht und effizient innerhalb einer umschriebenen Sitzungsanzahl durchführ- 
bar. Patienten berichten eine hohe Akzeptanz. Für Patienten ist das Verfahren sehr symptomspezifisch und nachhaltig erlernbar. Insbesondere für vorwiegend somatisch- bzw. körperorientierte Patienten stellt die Herangehensweise und Veranschaulichung mittels Biofeedbacktherapie einen motivierenden Zugang dar. Selbstwirksames Verhalten und Behandlungserfolge werden schnell sichtbar und können das Commitment und die Adhäsion für ein Gesamtbehandlungsangebot erhöhen. Hinzu kommt, dass die zunehmende Entwicklung und Verfügbarkeit von portablen Biofeedbackgeräten die Anwendbarkeit und Generalisierungsmöglichkeiten auch außerhalb therapeutischer Institutionen deutlich verbessert.

Interessenkonflikt: Die Autoren geben an, dass kein Interessenkonflikt besteht.

\section{Literatur}

1 Weissacher E, Heuser J. Biofeedback. Die alternative Methode zur Behandlung von Schmerzen und psychosomatischen Beschwerden. Kreuzlingen/München: Heinrich Hugendubel Verlag; 2008

2 Schwartz MS, Olson RP. A Historical Perspective on the Field of Biofeedback and Applied Psychophysiology. In: Schwartz MS, Andrasik F, eds. Biofeedback: A Practitioner's Guide. 3. Aufl. New York: Guilford Press; 2003

3 Segrave K. Lie detectors: a social history. Jefferson, N.C.: McFarland; 2004

4 Sterman MB, Friar L. Suppression of seizures in an epileptic following sensorimotor EEG feedback training. Electroencephalogr Clin Neurophysiol 1972; 33: 89-95

5 Kaplan BJ. Biofeedback in epileptics: equivocal relationship of reinforced EEG frequency to seizure reduction. Epilepsia 1975; 16: $477-485$

6 Schoenberg PL, David AS. Biofeedback for psychiatric disorders: a systematic review. Appl Psychophysiol Biofeedback 2014; 39: 109-135

7 Rief W, Birbaumer N. Grundsätzliches zu Biofeedback. In: Rief W, Birbaumer N, Hrsg. Biofeedback Grundlagen, Indikationen, Kommunikation, praktisches Vorgehen in der Therapie. 3. Aufl. Stuttgart: Schattauer; 2010

8 Walschulewski-Floruß H, Miltner WHR, Haag G. Biofeedback. In: Linden M, Hautzinger M, Hrsg. Verhaltenstherapiemanual. Berlin: Springer; 2011: 97-102

9 Ray W, Raczynski J, Rogers T et al. Evaluation of Clinical Biofeedback. New York: Plenum Press; 1979

10 Schwartz NM, Schwartz MS. Definitions of Biofeedback and Applied Psychophysiology. In: Schwartz MS, Andrasik F, eds. Biofeedback: A Practitioner's Guide. 3. Aufl. New York: Guilford Press; 2003

11 Peek C. A Primer of Biofeedback Instrumentation. In: Schwartz MS, Andrasik F, eds. Biofeedback: A Practitioner's Guide. 3. Aufl. New York: Guilford Press; 2003

12 Neumann N, Strehl U, Birbaumer N. A Primer of Electroencephalographic Intrumentation. In: Schwartz MS, Andrasik F, eds. Biofeedback: A Practitioner's Guide. 3. Aufl. New York: Guilford Press; 2003

13 Monastra V. Clinical Applications of Electroencephalographic Biofeedback. In: Schwartz MS, Andrasik F, eds. Biofeedback: A Practitioner's Guide. 3. Aufl. New York: Guilford Press; 2003

14 Kropp P, Niederberger U. Biofeedback bei Kopfschmerzen. Schmerz 2010; 24: 279-288; Quiz 289

15 Holroyd KA, Penzien DB, Hursey KG et al. Change mechanisms in EMG biofeedback training: cognitive changes underlying improvements in tension headache. J Consult Clin Psychol 1984; 52: 1039-1053

16 Rains JC. Change mechanisms in EMG biofeedback training: cognitive changes underlying improvements in ten- sion headache. Headache 2008; 48: 735-736; Discussion 736-737

17 Wheat AL, Larkin KT. Biofeedback of heart rate variability and related physiology: a critical review. Appl Psychophysiol Biofeedback 2010; 35: 229-242

18 Glombiewski JA, Bernardy K, Hauser W. Efficacy of EMGand EEG-Biofeedback in Fibromyalgia Syndrome: A Meta-Analysis and a Systematic Review of Randomized Controlled Trials. Evid Based Complement Alternat Med 2013: $10.1155 / 2013 / 962741$

19 Nelson DV, Bennett RM, Barkhuizen A et al. Neurotherapy of fibromyalgia? Pain Med 2010; 11: 912 - 919

20 Lehrer PM, Vaschillo E, Vaschillo B et al. Biofeedback treatment for asthma. Chest 2004; 126: $352-361$

21 Kapitza KP, Passie T, Bernateck $M$ et al. First non-contingent respiratory biofeedback placebo versus contingent biofeedback in patients with chronic low back pain: a randomized, controlled, double-blind trial. Appl Psychophysiol Biofeedback 2010; 35: 207 - 217

22 Nestoriuc $Y$, Rief W, Martin A. Meta-analysis of biofeedback for tension-type headache: efficacy, specificity, and treatment moderators. J Consult Clin Psychol 2008; 76: $379-396$

23 Babu AS, Mathew E, Danda D et al. Management of patients with fibromyalgia using biofeedback: a randomized control trial. Indian J Med Sci 2007; 61: 455-461

24 Micoulaud-Franchi JA, Quiles C, Fond G et al. The covariation of independent and dependant variables in neurofeedback: a proposal framework to identify cognitive processes and brain activity variables. Conscious Cogn 2014; 26: $162-168$

25 Martin A, Rief W. Wie wirksam ist Biofeedback? Bern: Hogrefe; 2009

26 Haus K, Held C, Kowalski A et al. Praxisbuch Biofeedback und Neurofeedback. Berlin: Springer; 2013

27 Schwartz MS. Intake Decisions and Preparation of Patients for Therapy. In: Schwartz MS, Andrasik F, eds. Biofeedback: A Practitioner's Guide. 3. Aufl. New York: Guilford Press; 2003

28 Landman GW, Drion I, van Hateren KJ et al. Device-guided breathing as treatment for hypertension in type 2 diabetes mellitus: a randomized, double-blind, sham-controlled trial. JAMA 2013; 173: 1346-1350

29 Sulzer J, Haller S, Scharnowski F et al. Real-time fMRI neurofeedback: progress and challenges. Neuroimage 2013; 76: 386-399

30 Hawkinson JE, Ross AJ, Parthasarathy S et al. Quantification of adverse events associated with functional MRI scanning and with real-time fMRI-based training. Int J Behav Med 2012; 19: 372-381

31 Kravitz HM, Esty ML, Katz RS et al. Treatment of fibromyalgia syndrome using low-intensity neurofeedback with the flexyx neurotherapy system: a randomized controlled clinical trail. J Neurother 2006; 10: 41 - 58

32 Teufel M, Stephan K, Kowalski A et al. Impact of biofeedback on self-efficacy and stress reduction in obesity: a randomized controlled pilot study. Appl Psychophysiol Biofeedback 2013; 38: 177-184

33 Giardino ND, Chan L, Borson S. Combined heart rate variability and pulse oximetry biofeedback for chronic obstructive pulmonary disease: preliminary findings. Appl Psychophysiol Biofeedback 2004; 29: 121-133

34 Glombiewski JA, Hartwich-Tersek J, Rief W. Two psychological interventions are effective in severely disabled, chronic back pain patients: a randomised controlled trial. Int J Behav Med 2010; 17: 97 - 107

35 Choi SW, Chi SE, Chung SY et al. Is alpha wave neurofeedback effective with randomized clinical trials in depression? A pilot study. Neuropsychobiology 2011; 63: $43-$ 51

36 Siepmann M, Aykac V, Unterdorfer J et al. A pilot study on the effects of heart rate variability biofeedback in patients with depression and in healthy subjects. Appl Psychophysiol Biofeedback 2008; 33: 195-201

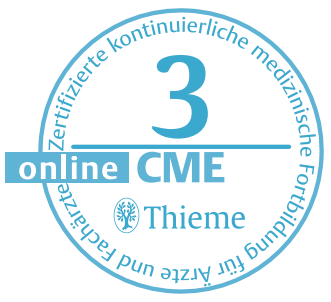


37 Mahtani KR, Nunan D, Heneghan Cl. Device-guided breathing exercises in the control of human blood pressure: systematic review and meta-analysis. J Hypertens 2012; 30: $852-860$

38 Vanathy S, Sharma PSVN, Kumar KB. The efficacy of alpha and theta neurofeedback training in treatment of generalized anxiety disorder. Indian J Clin Psychol 1998; 25: $136-143$

39 Mora MCS, Bleichhardt G, Weber D et al. Biofeedback bei kraniomandibulären Dysfunktionen. Psychotherapeut 2010; 55: 217 - 224

40 Nestoriuc $Y$, Martin A. Efficacy of biofeedback for migraine: a meta-analysis. Pain 2007; 128: 111 - 127

41 Kim S, Wollburg E, Roth WT. Opposing breathing therapies for panic disorder: a randomized controlled trial of lowering vs raising end-tidal $\mathrm{P}(\mathrm{CO}(2))$. J Clin Psychiatry 2012; 73: 931 - 939

42 Jacobs $S$, de Jong $A$. EMDR und Biofeedback in der Behandlung von posttraumatischen Belastungsstörungen. Ein neuropsychotherapeutisches Behandlungsprogramm. Göttingen: Hogrefe; 2007

43 Enck P, Van der Voort IR, Klosterhalfen S. Biofeedback therapy in fecal incontinence and constipation. Neurogastroenterol Motil 2009; 21: 1133-1141

44 Weise C, Heinecke K, Rief W. Biofeedback-based behavioral treatment for chronic tinnitus: results of a randomized controlled trial. J Consult Clin Psychol 2008; 76: 1046 1057

45 Burton AR, Rahman K, Kadota Y et al. Reduced heart rate variability predicts poor sleep quality in a case-control study of chronic fatigue syndrome. Exp Brain Res 2010; 204: $71-78$

46 Lehrer PM, Vaschillo E, Vaschillo B. Resonant frequency biofeedback training to increase cardiac variability: rationale and manual for training. Appl Psychophysiol Biofeedback 2000; 25: 177 - 191

47 Udupa K, Sathyaprabha TN, Thirthalli j et al. Alteration of cardiac autonomic functions in patients with major depression: a study using heart rate variability measures. J Affect Disord 2007; 100: 137-141

48 Wyller VB, Eriksen HR, Malterud K. Can sustained arousal explain the Chronic Fatigue Syndrome? Behav Brain Funct 2009; 5: 10

49 Lehrer PM, Vaschillo E, Vaschillo $B$ et al. Heart rate variability biofeedback increases baroreflex gain and peak expiratory flow. Psychosom Med 2003; 65: 796-805

50 Sherlin L, Gevirtz R, Wyckoff S et al. Effects of Respiratory Sinus Arrhythmia Biofeedback Versus Passive Biofeedback Control. Int J Stress Manag 2009; 16: 233-248

51 Rehfisch HP, Basler HD, Seemann H. Psychologische Schmerzbehandlung bei Rheuma. Berlin, Heidelberg: Springer; 1989

52 Thomas P, Grama C, Hiller W. Kognitive Verhaltenstherapie bei somatoformen Störungen. PID 2008; 9: 223 230

53 Martin A, Rief W. Somatoforme Störungen. In: Rief W, Birbaumer N, Hrsg. Biofeedback Grundlagen, Indikationen, Kommunikation, praktisches Vorgehen in der Therapie. 3. Aufl. Stuttgart: Schattauer; 2010

54 Barry RJ, Clarke AR, Johnstone SJ. A review of electrophysiology in attention-deficit/hyperactivity disorder: I. Qualitative and quantitative electroencephalography. Clin Neurophysiol 2003; 114: 171 - 183

55 Banaschewski T, Brandeis D. Neurophysiologie - elektrische Hirnaktivität. In: Steinhausen HC, Rothenberger A, Döpfner M, Hrsg. Handbuch ADHS Grundlagen, Klinik, Therapie und Verlauf der Aufmerksamkeitsdefizit-Hyperaktivitätsstörung. Stuttgart: Kohlhammer; 2010

56 Sergeant JA. Modeling attention-deficit/hyperactivity disorder: a critical appraisal of the cognitive-energetic model. Biol Psychiatry 2005; 57: 1248 - 1255

57 Arns M, de Ridder S, Strehl U et al. Efficacy of neurofeedback treatment in ADHD: the effects on inattention, im- pulsivity and hyperactivity: a meta-analysis. Clin EEG Neurosci 2009; 40: 180-189

58 Mayer K, Wyckoff SN, Strehl U. One size fits all? Slow cortical potentials neurofeedback: a review. J Atten Disord 2013; 17: 393-409

59 Tan G, Thornby J, Hammond DC et al. Meta-analysis of EEG biofeedback in treating epilepsy. Clin EEG Neurosci 2009; 40: 173-179

60 Morin CM, Hauri PJ, Espie CA et al. Nonpharmacologic treatment of chronic insomnia. An American Academy of Sleep Medicine review. Sleep 1999; 22: 1134-1156

61 Weiskopf N, Mathiak K, Bock SW et al. Principles of a braincomputer interface $(\mathrm{BCl})$ based on real-time functional magnetic resonance imaging (fMRI). IEEE Trans Biomed Eng 2004; 51: 966 - 970

62 Rota $G$, Sitaram $R$, Veit $R$ et al. Self-regulation of regional cortical activity using real-time fMRI: the right inferior frontal gyrus and linguistic processing. Hum Brain Mapp 2009; 30: 1605-1614

63 Caria A, Sitaram $R$, Veit $R$ et al. Volitional control of anterior insula activity modulates the response to aversive stimuli. A real-time functional magnetic resonance imaging study. Biol Psychiatry 2010; 68: 425-432

64 Shibata K, Watanabe T, Sasaki Y et al. Perceptual learning incepted by decoded fMRI neurofeedback without stimulus presentation. Science 2011; 334: 1413-1415

65 Scharnowski F, Hutton C, Josephs $O$ et al. Improving visual perception through neurofeedback. J Neurosci 2012; 32: $17830-17841$

66 deCharms RC, Maeda F, Glover GH et al. Control over brain activation and pain learned by using real-time functional MRI. Proc Natl Acad Sci U S A 2005; 102: 18626-18631

67 Haller S, Birbaumer N, Veit R. Real-time fMRI feedback training may improve chronic tinnitus. Eur Radiol 2010; 20: $696-703$

68 Subramanian L, Hindle $\mathrm{V}$, Johnston S et al. Real-time functional magnetic resonance imaging neurofeedback for treatment of Parkinson's disease. J Neurosci 2011 31: $16309-16317$

69 Li X, Hartwell KJ, Borckardt J et al. Volitional reduction of anterior cingulate cortex activity produces decreased cue craving in smoking cessation: a preliminary realtime fMRI study. Addict Biol 2013; 18: 739 - 748

70 Linden DE, Habes I, Johnston SI et al. Real-time self-regulation of emotion networks in patients with depression. PloS One 2012; 7: e38115

71 Ehlis AC, Schneider S, Dresler $T$ et al. Application of functional near-infrared spectroscopy in psychiatry. Neuroimage 2014; 85 Pt 1: 478-488

72 Mihara $M$, Hattori $N$, Hatakenaka $M$ et al. Near-infrared spectroscopy-mediated neurofeedback enhances efficacy of motor imagery-based training in poststroke victims: a pilot study. Stroke 2013; 44: 1091 - 1098

73 Barcala L, Grecco LA, Colella F et al. Visual biofeedback balance training using wii fit after stroke: a randomized controlled trial. J Phys Ther Sci 2013; 25: 1027 - 1032

74 Ryan M, Gevirtz R. Biofeedback-based psychophysiological treatment in a primary care setting: an initial feasibility study. Appl Psychophysiol Biofeedback 2004; 29: $79-93$

75 Sonuga-Barke EJ, Brandeis D, Cortese S et al. Nonpharmacological interventions for ADHD: systematic review and meta-analyses of randomized controlled trials of dietary and psychological treatments. Am J Psychiatry 2013; 170: $275-289$

76 Margraf J, Schneider S. Panik: Angstanfälle und ihre Behandlung. Berlin: Springer-Verlag; 1990

77 Kroymann R. Angststörungen. In: Rief W, Birbaumer N, Hrsg. Biofeedback Grundlagen, Indikationen, Kommunikation, praktisches Vorgehen in der Therapie. 3. Aufl. Stuttgart: Schattauer; 2010 


\section{CME-Fragen Biofeedback und Neurofeedback}

1 Welche Aussage ist richtig? Biofeedback ist ein Verfahren, das

A den tiefenpsychologisch psychotherapeutischen Behandlungsansätzen zugeordnet wird.

B dem Therapeuten die aktivere Rolle zuschreibt.

C das Erlernen neuer Verhaltensweisen ermöglicht.

D der Verbesserung der Interozeption wenig Beachtung schenkt.

E mit negativer Verstärkung arbeitet.

2 Welche Aussage ist richtig? Das primäre, unspezifische Ziel einer Biofeedback-Behandlung ist am ehesten die Erhöhung der externen Steuerung. die Verbesserung des Selbstwirksamkeitserlebens. das Erleben positiver Verstärkung. das Erleben von Entspannung. die Ablenkung von Beschwerden.

3 Welche Aussage ist richtig?

A Die Rückmeldung bei einer Biofeedbackbehandlung erfolgt vornehmlich haptisch.

B Die Rückmeldung bei einer Biofeedbackbehandlung dient hauptsächlich dem Therapeuten zur Erfolgskontrolle über den Behandlungsverlauf.

C In der Regel werden dem Patienten alle erhobenen Parameter parallel zurückgemeldet.

D Das Biofeedback wird selten in Kombination mit anderen Behandlungsverfahren eingesetzt.

E Meistens erfolgt die Auswahl des Trainings-Parameters symptomspezifisch.

\section{Welche Aussage ist richtig?}

A Das eigenständige Üben des Patienten über die Behandlungssitzungen hinaus ist mitentscheidend für den Behandlungserfolg.

B Die 1. Sitzung einer Biofeedbackbehandlung unterscheidet sich in der Regel nicht von den folgenden Sitzungen.

C Die Biofeedbackbehandlung stellt für Kinder eine zu anspruchsvolle Behandlung dar und wird daher nur bei Jugendlichen und Erwachsenen angewendet.

D Die Behandlung von Migräne und Spannungskopfschmerz mit Biofeedback erfolgt primär über EEG-Feedback.

E Bei unspezifischen körperlichen Beschwerden ohne organpathologisches Korrelat sollte das Biofeedback nicht eingesetzt werden.
5 Welche Aussage hinsichtlich der Forschung zum Biofeedback und der Wirksamkeit der Methode ist falsch?

A Der Einsatz von randomisierten kontrollierten Studien zur Überprüfung der Wirksamkeit von Biofeedback- und Neurofeedbackbehandlungen sollte weiterhin verstärkt werden.

B Biofeedback- und Neurofeedbackbehandlungen sollten im Vergleich zu anderen bestehenden Therapieansätzen beurteilt werden.

c Zur Beurteilung der Wirksamkeit einer spezifischen Biofeedbackbehandlung bei Essstörungen oder posttraumatischer Belastungsstörung liegen derzeit keine ausreichenden Daten vor.

D Der Einsatz der Neurofeedbackbehandlung bei Patienten mit Persönlichkeitsstörungen befindet sich noch im experimentellen Status.

E Bei der Behandlung von Angsterkrankungen stellt die Biofeedbacktherapie keine ergänzende Behandlungsoption dar.

6 Der Prozess der operanten Konditionierung spielt eine wichtige Rolle beim Erwerb der Selbstkontrolle von Hirnaktivität. Welche Aussage ist am ehesten zutreffend?

A Eine verzögerte Rückmeldung der Hirnaktivität beschleunigt den Lernvorgang.

B Die Selbstkontrolle ist nicht mit den Umgebungsreizen im Labor assoziiert und kann von allein in den Alltag generalisiert werden.

C Der Erfolg des Neurofeedback-Trainings ist unabhängig von der Motivation.

D Die Rückmeldung der Hirnaktivität wirkt als negativer Verstärker.

E Die erfolgreiche Selbstkontrolle der Hirnaktivität führt zu spezifischen Verhaltensänderungen.

7 Welche EEG-Kenngröße eignet sich besonders für eine Behandlung von ADHS?

Verringerung der Aktivität im Delta-Band

Verstärkung der Aktivität im Beta-Band

Verstärkung der Aktivität im Theta-Band

Erhöhung der P300-Amplitude

E Verringerung der Aktivität im Alpha-Band

8 Welche Aussage im Vergleich der verschiedenen Neurofeedbackverfahren ist am ehesten zutreffend? NIRS-Neurofeedback kann für das Training der Aktivität subkortikaler Strukturen verwendet werden.

B NIRS-Neurofeedback ist ein etabliertes klinisches Verfahren.

C EEG hat eine geringere räumliche Auflösung als fMRI.

D EEG-Neurofeedback eignet sich besonders für die Behandlung von Persönlichkeitsstörungen.

E fMRI-Neurofeedback ermöglicht die direkte Erfassung neuronaler Aktivität. 
9

A Die Behandlung von Migräne und Spannungskopfschmerzen unterscheiden sich im Biofeedback nicht.

B Feedback zu zentralen Parametern erfordert einen geringeren Sitzungsumfang als Feedback zu peripheren Parametern.

C Das Fokussieren auf Symptomveränderungen wird durch gezielte Biofeedbackbehandlung reduziert.

D Kognitive therapeutische Elemente sind bei Biofeedbackbehandlungen als übendem Verfahren nicht bedeutsam.

E Harn- und Stuhlinkontinenz werden u. a. mittels EMGTraining behandelt.

\section{0}

A Die therapeutische Beziehung ist im Rahmen einer Biofeedbackbehandlung unerheblich.

B Einzelne Behandlungsangebote in einem multimodalen psychosomatischen Behandlungssetting sind mit Biofeedbackbehandlungen nicht vereinbar.

C Biofeedback wird als ärztliche Kassenleistung angeboten.

D Die Generalisierung in den Alltag spielt bei Biofeedback eine nachrangige Rolle.

E Biofeedback bei somatoformen Störungen hat Einfluss auf Kognition, Emotion, Körper und Verhalten.

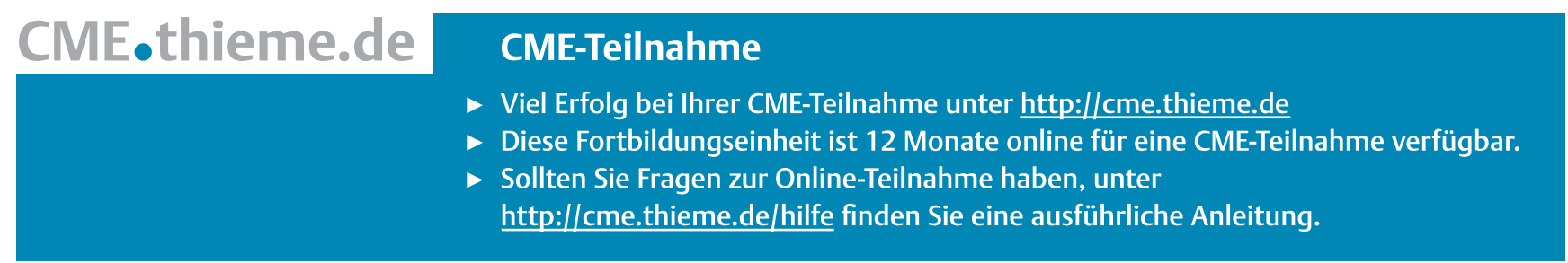

